\title{
ON METRIC REGULARITY IN METRIC SPACES
}

\author{
Serge Gautier and Karine Pichard
}

\begin{abstract}
We prove metric regularity results for both single-valued maps and set-valued maps defined between metric spaces.
\end{abstract}

\section{INTRODUCTION}

Since Graves' theorem (see [8]), many papers have been devoted to inversion theorems, open mapping principles and metric regularity problems. We are especially interested in metric regularity. Some results are obtained for single-valued maps (among others $[5,17])$ and others for set-valued maps (among others $[3,5,9,11,12,13,14$, $15,16,17]$ and references therein). However, in most cases, they are obtained for maps defined beetwen two vector spaces and the linear structure seems to play a key role although the notion of metric regularity is a concept which does not a priori require a linear structure. A natural question is how to extend these results in metric spaces. In $[6,7]$, some inverse mapping theorems are stated in this case. In $[4,10]$, metric regularity results are proved using a notion of slope.

The aim of this paper is to state metric regularity results for both single-valued and set-valued maps defined between metric spaces using the approach of mutational calculus, as in [2]. Each metric space is endowed with a mutational structure which allows a kind of differential calculus (see $[\mathbf{1}, \mathbf{2}, \mathbf{1 8}, \mathbf{1 9}])$. To get metric regularity results, it is necessary to make some assumptions on the target space of the function. The assumption made by Aubin in [2] in order to obtain a metric regularity result in the mutational case seems to be strong. In particular, we exhibit a simple mutational space for which it is not satisfied. The goal of this paper is, among other things, to weaken this hypothesis. The set-valued case is also studied.

In Part 2, we recall some notions we shall use throughout the paper. In Part 3, we prove a metric regularity result for a single-valued map. In Part 4 , we consider the case of a set-valued map.

\section{Received 22nd October, 2002}

Copyright Clearance Centre, Inc. Serial-fee code: 0004-9727/03 \$A2.00+0.00. 


\section{Preliminaries}

Let us consider two metric spaces, $X$ and $Y$, whose distances are respectively denoted by $d_{X}$ and $d_{Y}$ and open balls by $B_{X}$ and $B_{Y}$. If there is no possible confusion, we simply write $d$ and $B$. If $A$ is a subset of $X$, the distance $d_{X}(x, A)$ from $x$ to $A$ is defined as follows: $d_{X}(x, A)=\inf \left\{d_{X}(x, a): a \in A\right\}$.

The mutational calculus is a theory of differential calculus in metric spaces due to J.P. Aubin ([1]). The basic idea is to endow a metric space with a net of "directions".

We recall from [2] the following notion (a slightly different notion is considered in [1]):

Definition 2.1: A continuous application $u: X \times[0,1] \rightarrow X$ is said to be a transition if

(i) $\forall x \in X, u(x, 0)=x$

(ii) $\forall x \in X, \forall t \in\left[0,1\left[, \lim _{h \rightarrow 0^{+}}\left(d_{X}(u(x, t+h), u(u(x, t), h))\right) / h=0\right.\right.$

(iii) $\alpha(u)=\max \left(0, \sup _{x \neq y} \lim _{h \rightarrow 0^{+}}\left(d_{X}(u(x, h), u(y, h))-d_{X}(x, y)\right) /\left(h d_{X}(x, y)\right)\right)$ $<+\infty$

(iv) $\beta(u)=\sup _{x \in X} \limsup _{h \rightarrow 0^{+}}\left(d_{X}(u(x, h), x)\right) / h<+\infty$.

The space of all the transitions is denoted by $\mathcal{U}$. We now endow $\mathcal{U}$ with the following distance:

$$
d_{\Delta}(u, v)=\sup _{x \in X} \limsup _{h \rightarrow 0^{+}} \frac{d_{X}(u(x, h), v(x, h))}{h} .
$$

Let $\mathcal{D}$ be a nonempty subset of $\mathcal{U} .(X, \mathcal{D})$ is a mutational space if $\mathcal{D}$ is a closed subset of $\mathcal{U}$ and if $\mathcal{D}$ contains the neutral transition 1 , defined by $\mathbf{1}(x, h)=x, \forall x \in X, \forall h \in[0,1]$.

EXAMPLE 2.2. It is possible to construct a mutational space on the space of the compact sets of $\mathbb{R}^{n}$ (endowed with the Hausdorff distance). Let $M$ be a non negative real. We denote by $X_{M}$ the space of the compact sets of $\mathbb{R}^{n}$ which are included in $M B_{\mathbb{R}^{n}}$ and by $X_{M C}$ the points of $X_{M}$ that are convex. Let $C$ be in $X_{M C}$. We define a transition by the following way: $u_{C}: X_{M} \times[0,1] \rightarrow X_{M},(A, h) \mapsto e^{-h} A+\left(1-e^{-h}\right) C$. Any transition is generated by a convex set. Set $\mathcal{D}_{X_{M}}=\left\{u_{C}: C \in X_{M C}\right\}$. Then, $\left(X_{M}, \mathcal{D}_{X_{M}}\right)$ is a mutational space. We refer to $[\mathbf{1 9}, \mathbf{1 8}]$ for details.

Definition 2.3: Let $\left(X, \mathcal{D}_{X}\right)$ and $\left(Y, \mathcal{D}_{Y}\right)$ be two mutational spaces and $f: X$ $\rightarrow Y$. We say that $f$ is mutable in $x$ in the direction $u \in \mathcal{D}_{X}$, if there exists $v \in \mathcal{D}_{Y}$ such that

$$
\lim _{h \rightarrow 0^{+}} \frac{d_{Y}(f(u(x, h)), v(f(x), h))}{h}=0 .
$$

This is denoted by $v \in \stackrel{\circ}{f}(x) u$. We say that $f$ is strictly mutable at $x$ in the direction $u$ if there exists $v$ such that

$$
\lim _{h \rightarrow 0^{+}, x^{\prime} \rightarrow x} \frac{d Y\left(f\left(u\left(x^{\prime}, h\right)\right), v\left(f\left(x^{\prime}\right), h\right)\right)}{h}=0 .
$$


REMARK 2.4. If $f$ is a function into $X_{M}$, endowed with the space of transitions constructed in Example 2.2, the mutational derivative is unique so that you can simply write: $v=\stackrel{\circ}{f}(x) u$ (see [18]).

It is clear that the choice of the net of transitions is crucial. Indeed if it is poor, you can not hope to get good results. In the following definition, we introduce a notion of richness of a mutational space.

Definition 2.5: Let $\left(X, \mathcal{D}_{X}\right)$ be a mutational space. It is said to be rich at $x$ if the following condition is satisfied: there exists a function $k:[0,1] \rightarrow[0,1]$ increasing, equal to 0 at 0 , there exists $\mu>0$ such that, for any $y, z$ in $B(x, \mu)$, there exists $u$ in $\mathcal{D}_{X}$ satisfying:

$$
d_{X}(u(y, h), z) \leqslant(1-k(h)) d_{X}(y, z)
$$

for all $h$ in $[0,1]$.

EXAMPLE 2.6.

(1) Consider the case where $X$ is a normed vector space; a mutational space is simply constructed by using the following natural transitions: $u(x, h)$ $=x+h \bar{u}$, where $\bar{u}$ belongs to $X$. This mutational space is rich at any point $x$ : consider $k(h)=h$ and $u(t, h)=t+h(z-y)$.

(2) The space $\left(X_{M C}, \mathcal{D}_{X_{M C}}\right)$ is rich at any point. In this case, it is sufficient to consider the function $k(h)=1-e^{-h}$ and $u(t, h)=e^{-h} t+\left(1-e^{-h}\right) z$.

Let us now recall a last definition.

DEFINITION 2.7:

(i) A function $f: X \rightarrow Y$ is said to be metrically regular at $x_{0}$ if there exist $K>0, R>0$ and $r>0$ such that

$$
d_{X}\left(x, f^{-1}(y)\right) \leqslant K d_{Y}(f(x), y),
$$

for any $(x, y)$ in $B_{X}\left(x_{0}, r\right) \times B_{Y}\left(f\left(x_{0}\right), R\right)$.

(ii) A set-valued map $F: X \rightrightarrows Y$ is said to be metrically regular at a point $\left(x_{0}, y_{0}\right)$ of its graph if there exist $K>0, R>0$ and $r>0$ such that

$$
d_{X}\left(x, F^{-1}(y)\right) \leqslant K d_{Y}(F(x), y)
$$

for any $x$ in $B_{X}\left(x_{0}, \dot{r}\right)$, for any $y$ in $B_{Y}\left(y_{0}, R\right)$.

\section{The Single-VAlued CASE}

In this part, we consider the case of a single-valued map. Let us first recall a result of Aubin (see [2]). 
THEOREM 3.1. Let $\left(X, \mathcal{D}_{X}\right)$ and $\left(Y, \mathcal{D}_{Y}\right)$ be two mutational spaces and $f: X$ $\rightarrow Y$. We assume that:

(i) For any $y, z$ in $Y$ there exists $u$ in $\mathcal{D}_{Y}$ such that:

$$
\lim _{h \rightarrow 0^{+}} \frac{d_{Y}(u(z, h), y)-(1-h) d_{Y}(z, y)}{h}=0
$$

and $\beta(u) \leqslant d_{Y}(y, z)$.

(ii) There exists $c>0, \gamma>0$ such that $f$ is strictly mutable on the ball $B\left(x_{0}, \gamma\right)$ and the mutations $f(x)$ are surjective, and for every $x$ in $B\left(x_{0}, \gamma\right)$, for any $w$ in $\mathcal{D}_{Y}$ there exists $v$ in $\mathcal{D}_{X}$ such that $w \in \stackrel{\circ}{f}(x) v$ and $\beta(v) \leqslant \beta(w)$.

Then, there exists $l>0$ such that for any $y$ in $\operatorname{int}\left(B\left(f\left(x_{0}\right),(\gamma / c)\right)\right)$, there exists a solution $\bar{x}$ to the equation $f(x)=y$ satisfying $d_{X}\left(\bar{x}, x_{0}\right) \leqslant l d_{Y}\left(y, f\left(x_{0}\right)\right)$.

REMARK 3.2. It is quite easy to see that the assumption (i) is not satisfied for the mutational space $\left(X_{M C}, \mathcal{D}_{X_{M C}}\right)$ : Let $Z$ and $Y$ be two points of $X_{M C}$. For the equality to be satisfied, we necessary have $u(X, h)=e^{-h} X+\left(1-e^{-h}\right) Y$. Then, $h^{-1} d_{H}(u(X, h), X)=h^{-1}\left(1-e^{-h}\right) d_{H}(X, Y)$, this shows that the second condition is not satisfied. When looking more precisely, we realise that the required inequality in (i) is in fact an equality, this explains why this assumption is difficult to satisfy: Using the triangular inequality and the first condition of (i), we have $h^{-1} d_{Y}(u(z, h), z)$ $\geqslant d_{Y}(z, y)+\varepsilon(h)$ with $\lim _{h \rightarrow 0^{+}} \varepsilon(h)=0$. Consequently, $\liminf _{h \rightarrow 0^{+}} h^{-1} d_{Y}(u(z, h), z) \geqslant d_{Y}(z, y)$. Using the inequality of $(\mathrm{i})$, we deduce that $\beta(u)=d_{Y}(z, y)$.

Let us now state the main result of this part.

THEOREM 3.3. Let $\left(X, \mathcal{D}_{X}\right)$ and $\left(Y, \mathcal{D}_{Y}\right)$ be two mutational spaces, $X$ being complete. Let $f: X \rightarrow Y$ be a continuous function: Let a be in $X$. We suppose that $\left(Y, \mathcal{D}_{Y}\right)$ is rich at $f(a)$. We assume that:

(h1) $\exists c>0 \exists \beta>0 \forall x \in B_{X}(a, \beta), \forall v \in \mathcal{D}_{Y}, \exists u \in \mathcal{D}_{X}: v \in \stackrel{\circ}{f}(x) u$, and

$$
d_{X}(u(x, h), x) \leqslant c d_{Y}(f(x), v(f(x), h))
$$

for all $h$ in $[0,1]$

(h2) $\forall \varepsilon>0 \exists \alpha>0 \exists \eta>0 \forall x \in B_{X}(a, \alpha) \forall u \in \mathcal{D}_{X} \forall v \in \stackrel{\circ}{f}(x) u$ :

$$
d_{Y}(f(u(x, h)), v(f(x), h)) \leqslant \varepsilon d_{X}(u(x, h), x),
$$

for all $h$ in $[0, \eta]$.

(h3) $\exists \varepsilon>0$ :

$$
k(\eta)>\frac{2 \varepsilon c}{1+\varepsilon c}
$$


where $c$ is defined by (h1), $\eta$ by (h2), and $k$ by the assumption of richness of $\left(Y, \mathcal{D}_{Y}\right)$.

Then, $f$ is metrically regular at $a$.

Proof: Let $\alpha, \beta, \eta, \varepsilon$ be the constants defined by the assumptions (h1), (h2), (h3) and $\mu$ defined by Definition 2.5. Set $\gamma=2 \varepsilon c+1-k(\eta)(1+\varepsilon c)$. Observe that $0<\gamma<1$. Set

$$
R=\min \left(\frac{(1-\gamma) \min (\alpha, \beta)}{4 c(2-k(\eta))}, \frac{\mu}{4}\right)
$$

The function $f$ is continuous at $a$ : there exists $\zeta$ such that for all $x$ in $B_{X}(a, \zeta), f(x)$ belongs to $B_{Y}(f(a), R)$. Set $r=\min (\zeta,(\alpha / 2),(\beta / 2))$. Let $x$ be in $B_{X}(a, r)$ and $y$ be in $B_{Y}(f(a), R)$. We have:

$$
d_{Y}(f(x), y) \leqslant \min \left(\frac{(1-\gamma) \min (\alpha, \beta)}{2 c(2-k(\eta))}, \frac{\mu}{2}\right) .
$$

We are going to construct a sequence $\left(x_{n}, y_{n}\right)_{n \in \mathbb{N}}$ such that

(i) $y_{n}=f\left(x_{n}\right)$

(ii) $x_{n} \in B_{X}(a, \min (\alpha, \beta))$

(iii) $d_{Y}\left(y_{n}, y\right) \leqslant \gamma^{n} d_{Y}(y, f(x))$

(iv) $d_{X}\left(x_{n+1}, x_{n}\right) \leqslant(2-k(\eta)) c \gamma^{n} d_{Y}(y, f(x))$

(v) $y_{n} \in B_{Y}(f(a), \mu)$.

The sequence begins with $\left(x_{0}, y_{0}\right)=(x, f(x))$. Assume that we have already constructed $\left(x_{i}, y_{i}\right)$ for $i=1, \ldots, n$. We now construct $\left(x_{n+1}, y_{n+1}\right)$. The space $\left(Y, \mathcal{D}_{Y}\right)$ is rich at $f(a)$, then there exists $v$ in $\mathcal{D}_{Y}$ such that:

$$
d_{Y}\left(v\left(y_{n}, \eta\right), y\right) \leqslant(1-k(\eta)) d_{Y}\left(y, y_{n}\right) .
$$

Let us now apply the assumption (h1) to $x_{n}, \eta$ and $v$ : there exists $u$ in $\mathcal{D}_{X}$ such that $v \in \stackrel{\circ}{f}(x) u$ and

$$
d_{X}\left(u\left(x_{n}, \eta\right), x_{n}\right) \leqslant c d_{Y}\left(f\left(x_{n}\right), v\left(f\left(x_{n}\right), \eta\right)\right)
$$

We then use (h2):

$$
d_{Y}\left(f\left(u\left(x_{n}, \eta\right)\right), v\left(f\left(x_{n}\right), \eta\right)\right) \leqslant \varepsilon d_{X}\left(u\left(x_{n}, \eta\right), x_{n}\right) .
$$

We set $x_{n+1}=u\left(x_{n}, \eta\right)$ and $y_{n+1}=f\left(x_{n+1}\right)$. Using the triangular inequality and (1), we get

$$
d_{Y}\left(y_{n}, v\left(y_{n}, \eta\right)\right) \leqslant(2-k(\eta)) d_{Y}\left(y_{n}, y\right)
$$

On the other hand, using (2) and (3), we get

$$
d_{Y}\left(y_{n+1}, v\left(y_{n}, \eta\right)\right) \leqslant \varepsilon c d_{Y}\left(y_{n}, v\left(y_{n}, \eta\right)\right)
$$


We then obtain:

$$
d_{Y}\left(y_{n+1}, v\left(y_{n}, \eta\right)\right) \leqslant \varepsilon c(2-k(\eta)) d_{Y}\left(y_{n}, y\right) .
$$

From the previous inequality and the estimate (1), we deduce:

$$
d_{Y}\left(y_{n+1}, y\right) \leqslant d_{Y}\left(y_{n+1}, v\left(y_{n}, \eta\right)\right)+d_{Y}\left(v\left(y_{n}, \eta\right), y_{n}\right) \leqslant \gamma d_{Y}\left(y_{n}, y\right)
$$

This yields to

$$
d_{Y}\left(y_{n+1}, y\right) \leqslant \gamma^{n+1} d_{Y}(f(x), y)
$$

We also have:

$$
d_{Y}\left(y_{n+1}, f(a)\right) \leqslant d_{Y}\left(y_{n+1}, y\right)+d_{Y}(y, f(a))<\mu .
$$

From (2) and (4), we first get $d_{X}\left(x_{n+1}, x_{n}\right) \leqslant c(2-k(\eta)) d_{Y}\left(y_{n}, y\right)$ and then, with the help of (iii),

$$
d_{X}\left(x_{n+1}, x_{n}\right) \leqslant(2-k(\eta)) c \gamma^{n} d_{Y}(f(x), y)
$$

We have

$$
d_{X}\left(x_{n+1}, a\right) \leqslant(2-k(\eta)) c \frac{1-\gamma^{n}}{1-\gamma} d_{Y}(f(x), y)+d_{X}(x, a)
$$

and then

$$
d_{X}\left(x_{n+1}, a\right) \leqslant(2-k(\eta)) c \frac{1}{1-\gamma}\left(d_{Y}(f(x), f(a))+d_{Y}(f(a), y)\right)+\frac{1}{2} \min (\alpha, \beta) .
$$

This leads to

$$
d_{X}\left(x_{n+1}, a\right) \leqslant \min (\alpha, \beta) .
$$

In consequence, $\left(x_{n+1}, y_{n+1}\right)$ satisfies the five required conditions. From inequality (iii) (and the fact that $0<\gamma<1$ ), it is easy to see that the sequence $\left(y_{n}\right)_{n \in N}$ converges to $y$. On the other hand, a simple verification leads to

$$
d_{X}\left(x_{n+p}, x_{n}\right) \leqslant \gamma^{n}(2-k(\eta)) c d_{Y}(f(x), y) \frac{1-\gamma^{p}}{1-\gamma} .
$$

The sequence $\left(x_{n}\right)_{n \in \mathbb{N}}$ is a Cauchy sequence in a complete metric space, so it converges to a point $\bar{x}$ of $X$. This point satisfies $y=f(\bar{x})$. We also have:

$$
d_{X}\left(x_{n}, x\right) \leqslant(2-k(\eta)) c \frac{1-\gamma^{n}}{1-\gamma} d_{Y}(f(x), y)
$$

Therefore,

$$
d_{X}(\bar{x}, x) \leqslant(2-k(\eta)) c \frac{1}{1-\gamma} d_{Y}(f(x), y)
$$

The result follows with $K=((2-k(\eta)) c) /(1-\gamma)$. 
REMARK 3.4. If $Y$ is a normed vector space, (h3) can be dropped. Choose $\varepsilon>0$ such that $\varepsilon c<1$. It is possible to take $\eta<(1 / 2)$ so that $(\varepsilon c+1) \eta<1$. Set $\gamma=(\varepsilon c+1) \eta$ and

$$
R=\frac{(1-\gamma) \min (\alpha, \beta)}{2 c \eta} \text {. }
$$

The proof is similar at the proof of Theorem 3.3.

REMARK 3.5. We can avoid (h3) if we strengthen the notion of richness of $\left(Y, \mathcal{D}_{Y}\right)$, by considering the following notion. The space $\left(Y, \mathcal{D}_{Y}\right)$ is said to be super-rich at $x$ if the following condition is satisfied. there exists a function $k:[0,1] \rightarrow[0,1]$ increasing, equal to 0 at 0 , there exists $\mu$, such that, for any $y, z$ in $B_{Y}(x, \mu)$, there exists $u$ in $\mathcal{D}_{Y}$ satisfying:

$$
d_{Y}(u(y, h), z) \leqslant(1-k(h)) d_{Y}(y, z) \text { and } d_{Y}(u(y, h), y) \leqslant k(h) d_{Y}(y, z)
$$

for all $h$ in $[0,1]$ (In particular, we have $d_{Y}(u(y, h), z)=(1-k(h)) d_{Y}(y, z)$ and $\left.d_{Y}(u(y, h), y)=k(h) d_{Y}(y, z)\right)$. In this case, we choose $\varepsilon>0$ such that $\varepsilon c<1$. Set $\gamma=1-k(\eta)+k(\eta) \varepsilon c$. The proof is similar at the proof of Theorem 3.3. This assumption is not comparable to the assumption (i) of Theorem 3.1 but the space $\left(X_{M C}, \mathcal{D}_{X_{M C}}\right)$ is super-rich at any point.

Let us now detail what is happening in the particular case of a set-valued map. Let $F: X \rightrightarrows \mathbb{R}^{n}$ be a set-valued map, with nonempty convex compact values; we denote by $f: X \rightarrow X_{M C}$ the single-valued map, defined by $F(x)=f(x)$. If $f$ is mutable in $x$ in the direction $u$, we denote by $\stackrel{\circ}{F}(x) u$ the unique convex compact set such that $\stackrel{\circ}{f}(x) u=e^{-h} f(x)+\left(1-e^{-h}\right) \stackrel{\circ}{F}(x) u$.

THEOREM 3.6. Let $(X, \mathcal{D})$ be a mutational space, $X$ being complete. We consider a set-valued map $F: X \rightrightarrows \mathbb{R}^{n}$ with nonempty, convex, compact values. We suppose that $F$ is continuous with respect to the Hausdorff distance, denoted by $d_{H}$. Let a be in $X$. Assume that:

(h1) $\exists c>0 \exists \beta>0 \exists M>0 \forall x \in B_{X}(a, \beta), \forall D \in B_{X_{M C}}(F(a), M), \exists u \in \mathcal{D}_{X}$ : $\stackrel{\circ}{F}(x) u=D$ and

$$
d_{X}(u(x, h), x) \leqslant c\left(1-e^{-h}\right) d_{H}(F(x), D),
$$

for all $h$ in $[0,1]$.

(h2) $\forall \varepsilon>0 \exists \alpha>0 \exists \eta>0 \forall x \in B_{X}(a, \alpha) \forall u \in \mathcal{D}_{X}$ such that $\stackrel{\circ}{F}(x) u$ $\in B_{X_{M C}}(F(a), M)$ :

$d_{H}\left(F(u(x, h)), e^{-h} F(x)+\left(1-e^{-h}\right) \stackrel{\circ}{F}(x) u\right) \leqslant \varepsilon d_{X}(u(x, h), x)$, for all $h$ in $[0, \eta]$. 
Then, there exists $r>0, R>0, K>0$ such that

$$
d_{X}\left(x, F^{-1}(y)\right) \leqslant K d_{H}(y, F(x)),
$$

for all $x$ in $B_{X}(a, r)$, for all $y$ such that $d(y, F(a)) \leqslant R$.

Proof: We consider the mutational space $X_{M C}$ introduced in Example 2.2. The single-valued map $f$ satisfies the assumptions of Thereom 3.3. Consequently, there exist $R>0, r>0, K>0$, such that

$$
d\left(x, f^{-1}(A)\right) \leqslant K d(f(x), A)
$$

for any $x$ in $B_{X}(a, r)$, for any $A$ in $B_{X_{M C}}(F(a), R)$. As $f$ is continuous, we can choose $r$ such that $d_{H}(f(x), f(a)) \leqslant(R / 3)$. Let $x$ be in $B_{X}(a, r)$ and $y$ be such that $d_{H}(y, F(a))$ $\leqslant(R / 3)$. We consider the set $A=\operatorname{co}(F(x) \cup\{y\})$ where co represents the closed convex hull. We have $d_{H}(F(x), A)=d_{\mathbb{R}^{n}}(y, F(x))$. Hence, from the triangular inequality, we get

$$
d_{H}(F(a), A) \leqslant d_{H}(F(a), F(x))+d_{H}(y, F(a))+d_{H}(F(a), F(x)),
$$

this leads to $d_{H}(F(a), A) \leqslant R$. We then obtain that $d_{X}\left(x, f^{-1}(A)\right) \leqslant K d_{H}(y, F(x))$. We have $f^{-1}(A) \subset F^{-1}(y)$. Indeed, if $x^{\prime}$ belongs to $f^{-1}(A)$, we have $f\left(x^{\prime}\right)=A ; y$ belongs to $A$, then $y \in F^{-1}\left(x^{\prime}\right)$ and then $x^{\prime}$ belongs to $F^{-1}(y)$. We conclude that

$$
d_{X}\left(x, F^{-1}(y) \leqslant d_{X}\left(x, f^{-1}(A)\right) \leqslant K d_{H}(y, F(x)),\right.
$$

which concludes the proof.

This result depends only on the point $a$ and not on a point $(a, b)$ of the graph of $F$. In the following part, we intend to localise the statement of Theorem 3.6.

\section{The set-valued Case}

In [3], a notion of differentiability (extended in [11]) is introduced for a set-valued map $F: X \rightrightarrows Y$, where $X, Y$ are normed vector spaces. In this section, we adapt this notion in the case where $X$ is only a metric space in order to obtain a metric regularity result.

Let $(X, \mathcal{D})$ be a mutational space and $Y$ be a normed vector space, $X$ being complete ( $d$ is the distance on $X$ and $\|$.$\| the norm on Y$ ).

DEFinition 4.1: We consider a set-valued map $F: X \rightrightarrows Y$. We say that $L$ is an approximation of $F$ at a point $(a, b)$ of $F$ if for any $\varepsilon>0$, there exists $r>0$ such that:

$$
L(u(x, h)) \cap B_{Y} \subset F(u(x, h))-z-\varepsilon d(u(x, h), x) B_{Y}
$$

for all $x$ in $B_{X}(a, r)$, for all $z$ in $B_{Y}(b, r) \cap F(x)$, for all $u$ in $\mathcal{D}$ and for all $h$ satisfying $d(u(x, h), x)<r$. 
TheOREM 4.2. We suppose that $X$ is complete. We consider a set-valued map $F: X \rightrightarrows Y$, with closed graph. Let $(a, b)$ be in $F$. Assume that:

(h1) $L$ is an approximation of $F$ at $(a, b)$.

(h2) $\exists \alpha>0 \exists c>0, \forall z \in \alpha B, \forall x \in B_{X}(a, r) \exists u \in \mathcal{D} \exists h>0$ :

$$
z \in L(u(x, h)) \text { and } d(u(x, h), x) \leqslant c\|z\|
$$

Then, there exist $R>0, K>0$ such that for all $y \in B_{Y}(b, R)$, there exixts $x$ in $B_{X}(a, r)$ satisfying

$$
y \in F(x) \text { and } d(x, a) \leqslant K\|y-b\| .
$$

Proof: Let $\varepsilon>0$ be chosen such that $\varepsilon c<1$. Set $R=\min (\alpha,(r / c),(r / c)(1$ $-\varepsilon c), 1)$. Set $y$ in $B(b, R)$. We are going to construct a sequence $\left(x_{n}, y_{n}\right)$ satisfying the following conditions:
(i) $x_{n} \in B_{X}(a, r)$
(ii) $y_{n} \in F\left(x_{n}\right) \cap B_{Y}(y, \alpha)$
(iii) $\left\|y-y_{n}\right\| \leqslant(\varepsilon c)^{n}\|y-b\|$
(iv) $d\left(x_{n-1}, x_{n}\right) \leqslant(\varepsilon c)^{n-1} c\|y-b\|$.

Assume that the elements $\left(x_{i}, y_{i}\right), i=1, \ldots, n$, have already been constructed. Let us build $\left(x_{n+1}, y_{n+1}\right)$. We first apply (h2) to $z=y-y_{n}$ and $x=x_{n}$. There exist $u \in \mathcal{D}$ and $t>0$ such that $y-y_{n} \in L\left(u\left(x_{n}, t\right)\right)$ and $d\left(u\left(x_{n}, t\right), x_{n}\right) \leqslant c\left\|y-y_{n}\right\|$. Set $x_{n+1}=u\left(x_{n}, t\right)$. We have

$$
d\left(x_{n+1}, x_{n}\right) \leqslant c(\varepsilon c)^{n}\|y-b\| .
$$

We now apply (h1) to $z=y_{n}, x_{n}$ and $u$. For all $h$ such that $d(u(x, h), x)<r$, we have

$$
L\left(u\left(x_{n}, h\right)\right) \cap B_{Y} \subset F\left(u\left(x_{n}, h\right)\right)-y_{n}-\varepsilon d\left(u\left(x_{n}, h\right), x_{n}\right) B_{Y} .
$$

Now, $d\left(u\left(x_{n}, t\right), x_{n}\right) \leqslant(\varepsilon c)^{n} c\|y-b\|$ and then $d\left(u\left(x_{n}, t\right), x_{n}\right) \leqslant(\varepsilon c)^{n} r<r$. So,

$$
L\left(x_{n+1}\right) \cap B_{Y} \subset F\left(x_{n+1}\right)-y_{n}-\varepsilon d\left(x_{n+1}, x_{n}\right) B_{Y} .
$$

There exist $y_{n+1}$ in $F\left(x_{n+1}\right)$ and $w$ in $B_{Y}$ such that

$$
y-y_{n}=y_{n+1}-y_{n}-\varepsilon d\left(x_{n+1}, x_{n}\right) w .
$$

We then obtain:

$$
\left\|y-y_{n+1}\right\| \leqslant(\varepsilon c)^{n+1}\|y-b\|
$$

and consequently $\left\|y-y_{n+1}\right\|<\alpha$. We have:

$$
d\left(x_{n+1}, a\right) \leqslant c\|y-b\| \frac{1-(\varepsilon c)^{n+1}}{1-\varepsilon c}
$$


this allows us to conclude that $x_{n+1}$ belongs to $B(a, r)$. The sequence $\left(y_{n}\right)_{n \in \mathbb{N}}$ converges to $y$ (condition (iii)). The sequence $\left(x_{n}\right)_{n \in \mathbb{N}}$ is a Cauchy sequence; indeed,

$$
d\left(x_{n+p}, x_{n}\right) \leqslant c\|y-b\|(\varepsilon c)_{n} \frac{1-(\varepsilon c)^{p-1}}{1-\varepsilon c} .
$$

In consequence, the sequence $\left(x_{n}\right)_{n \in \mathbb{N}}$ converges to a point $x$. As $F$ has a closed graph, $y$ belongs to $F(x)$ and we have:

$$
d(x, a) \leqslant \frac{c}{1-\varepsilon c}\|y-b\|
$$

this concludes the proof.

CoRollary 4.3. Under the assumptions of the theorem, we have: for all $\left(x_{1}, y_{1}\right)$ in $B((a, b),(r / 2))$, with $y_{1}$ in $F\left(x_{1}\right)$ for all $y$ in $B_{Y}\left(y_{1},(R / 2)\right)$ there exixts $x$ in $B_{X}\left(x_{1},(r / 2)\right)$ such that

$$
y \in F(x) \text { and } d\left(x, x_{1}\right) \leqslant K\left\|y-y_{1}\right\|,
$$

where $R, K$ and $r$ are the constants defined in Theorem 4.2.

Proof: The assumptions of Theorem 4.2 are satisfied with $\left(x_{1}, y_{1}\right)$ instead of $(a, b)$; the radius on which the assumptions are satisfied is now $r / 2$. Therefore, the estimate is obtained on a smallest neighbourhood of $y_{1}$.

LEMMA 4.4. Let $F$ be a set-valued map and $(a, b)$ be a point of its graph. Assume that the following condition is satisfied.

$$
\forall \varepsilon>0 \exists r>0: x \in B(a, r) \Rightarrow d(b, F(x))<\varepsilon .
$$

Let $\nu>0$. Then, there exists $\bar{r}$ such that for all $(x, y)$ in $B(a, \bar{r}) \times B(b, \bar{r})$ we have

$$
d(y, F(x))=d(y, F(x) \cap B(b, \nu)) .
$$

Proof: Let $\nu>0$. We apply Condition (5) to $\varepsilon=\nu / 4$. There exists $\bar{r}$ such that for $x$ in $B(a, \bar{r})$, we have $d(b, F(x))<\nu / 4$; this implies that there exists $\bar{z}$ in $F(x)$ such that $d(b, \bar{z}) \leqslant \nu / 4$. Let $y$ be in $B(b,(\nu / 4))$. Let $z$ not be in $B(b, \nu)$. We have:

$$
d(y, F(x)) \leqslant d(y, \bar{z}) \leqslant d(y, b)+d(b, \bar{z}),
$$

this leads to $d(y, F(x)) \leqslant \nu / 2$. We also have:

$$
d(y, z) \geqslant d(z, b)-d(y, b)
$$

and then

Hence,

$$
d(y, z) \geqslant \frac{3 \nu}{4}
$$

$$
d(y, z) \geqslant \frac{\nu}{2}+\frac{\nu}{4} \geqslant \frac{\nu}{4}+d(y, F(x)) .
$$

This is true for any $z \notin B(b, \nu)$. The conclusion holds. 
Corollary 4.5. Under the assumptions of the Theorem 4.2 and Condition (5), $F$ is metrically regular at $(a, b)$.

Proof: We apply Lemma 4.4 to $\nu=\min (r / 2, R / 4)$ and Corollary 4.3. Let $y$ be in $B_{Y}(b, \min (R / 4, \bar{r}))$ and $x$ be in $B_{X}(a, \min (r / 2, \bar{r}))$. Since the set $F(x)$ $\cap B_{Y}(b, \min (r / 2, R / 4))$ is nonempty, let us choose $y_{1}$ in this set. Observe that $\left(x, y_{1}\right)$ belongs to $B((a, b), r / 2)$ and $y$ belongs to $B_{Y}\left(y_{1}, R / 2\right)$. We can apply Corollary 4.3. There exists $x_{1}$ in $B_{X}(x, r / 2)$ (then in $B_{X}(a, r)$ ) such that $y$ belongs to $F\left(x_{1}\right)$ and $d_{X}\left(x, x_{1}\right) \leqslant K\left\|y-y_{1}\right\|$. Therefore,

$$
d\left(F^{-1}(y), x\right) \leqslant K d(y, F(x) \cap B(b, \min (r / 2, R / 4)))
$$

According to Lemma 4.4, with $\nu=\min (r / 2, R / 4)$, the result follows.

\section{REFERENCES}

[1] J.P. Aubin, 'Mutational equations in metric spaces', Set-valued Anal. 1 (1993), 3-46.

[2] J.P. Aubin, Mutational and morphological analysis. Tools for shape evolution and morphogenesis (Birkhäuser, Boston MA, 1999).

[3] D. Azé, 'An inversion theorem for set-valued maps', Bull. Austral. Math. Soc. 37 (1988), 411-414.

[4] D. Azé and J.N. Corvellec and R.E. Lucchetti, 'Variational pairs and applications to stability in nonsmooth analysis', Nonlinear Anal. 49 (2002), 643-670.

[5] J.M. Borwein and D.M. Zhuang, 'Verifiable necessary and sufficient conditions for openess and regularity of set-valued and single-valued maps', J. Math. Anal. Appl. 134 (1988), 441-459.

[6] H. Frankowska, 'Some inverse mapping theorems', Ann. Inst. Henri Poincaré Anal. Non Linéaire 7 (1990), 183-234.

[7] H. Frankowska, 'Conical inverse mapping theorems', Bull. Austral. Math. Soc. 45 (1992), 53-60.

[8] L.M. Graves, 'Some mapping theorems', Duke Math. 17 (1950), 111-114.

[9] A. Ioffe, 'On perturbation stability of metric regularity. Wellposedness in optimization and related topics', Set-Valued Anal. 9 (2001), 101-109.

[10] A. Ioffe, 'Towards metric theory of metric regularity', (Preprint of the University of Technion, Israël, 2002).

[11] A. Jourani, 'On metric regularity result', Bull. Austral. Math. Soc. 44 (1991), 1-9.

[12] A. Jourani and L.Thibault, 'Approximate subdifferential and metric regularity: the finite-dimensional case', Math. Programming 47 (1990), 203-218.

[13] A. Jourani and L.Thibault, 'Approximations and metric regularity in mathematical programming in Banach spaces', Math. Oper. Res. 18 (1993), 390-401.

[14] A. Jourani and L.Thibault, 'Metric regularity for strongly compactly Lipschitzian mappings', Nonlinear Anal. 24 (1995), 229-240.

[15] B. Mordukhovich, Complete characterization of openess, metric regularity and Lipschitzian properties of multifunctions, Trans. Amer. Math. Soc. 340 (1993), 1-35. 
[16] J.P. Penot, Metric regularity, openess and Lipschitzian behavior of multifunctions, Nonlinear Anal. 13 (1989), 629-643.

[17] J.P. Penot, 'Open mappings theorems and linearization stability', Numer. Funct. Anal. Optim. 8 (1985), 21-35.

[18] K. Pichard, Equations différentielles dans les espaces métriques. Applications à l'évolution de domaines, (Thèse) (Université de Pau, France, 2001).

[19] K. Pichard and S. Gautier, 'Equations with delay in metric spaces: the mutational approach', Numer. Funct. Anal. Optim. 7 and 8 (2000), 917-932.

Université de Pau

Laboratoire de Mathématiques

avenue de l'Université

$64000 \mathrm{Pau}$

France

e-mail: serge.gautier@univ-pau.fr

karine.pichard@univ-pau.fr 